Results In the first calendar 12 months of operation, the Melbourne MSU operated 30.5 service weeks and provided prehospital thrombolysis (tPA) to $\mathrm{n}=52$ patients (44\% of eligible infarcts) and directed $n=33$ patients for endovascular thrombectomy, of which $48 \%$ required bypass from the closest nonthrombectomy hospital. The overall median onset-to-tPA for MSU patients was 97.5 mins compared to the Australian metropolitan median of 150 mins. Thrombolysis in the first 'golden hour' increased to $13.5 \%$ from $3.3 \%$ in-hospital. Median onset-to-groin for MSU patients receiving EVT was 162 mins compared to 234 mins from historical controls.

Discussion Prehospital treatment and triage using the Mobile Stroke Unit in metropolitan Melbourne resulted in substantial improvements in commencement of reperfusion therapy. Workflow times are approximately halved for thrombolysis and endovascular thrombectomy respectively. Prehospital thrombolysis also allowed a $>400 \%$ increase in the proportion of treatment in the first 'golden hour'.

\section{MECHANICAL THROMBECTOMY IN PEDIATRIC STROKE: SYSTEMATIC REVIEW, INDIVIDUAL PATIENT-DATA META-ANALYSIS, AND CASE SERIES}

\begin{abstract}
${ }^{1,2}$ Kartik Bhatia, ${ }^{1}$ Hans Kortman, ${ }^{3,4}$ Chris Blair*, ${ }^{2}$ Geoffrey Parker, ${ }^{2}$ David Brunacci, ${ }^{4,2} \mathrm{Tim}$ Ang, ${ }^{5}$ John Worthington, ${ }^{6}$ Prakash Muthusami, ${ }^{1}$ Timo Krings. ${ }^{1}$ Department of Interventional Neuroradiology, Toronto Western Hospital, Toronto, Ontario, Canada; ${ }^{2}$ Department of Interventional Neuroradiology, Royal Prince Alfred Hospital, Sydney, NSW, Australia; ${ }^{3}$ Department of Neurology, Liverpool Hospital, Liverpool, NSW, Australia; ${ }^{4}$ Department of Neurology, Royal Prince Alfred Hospital, Camperdown, NSW, Australia; ${ }^{5}$ Department of Neurology, Royal Prince Alfred Hospital, Sydney, NSW, Australia; ${ }^{6}$ Department of Interventional Radiology, The Hospital for Sick Children, Toronto, Ontario, Canada
\end{abstract}

\subsection{6/jnnp-2019-anzan.12}

Introduction In adults, there is strong evidence demonstrating the superiority of mechanical thrombectomy (MT) plus intravenous thrombolysis over thrombolysis alone for the treatment of acute ischemic stroke due to large vessel occlusion (LVO). The role of MT in the paediatric stroke population is less clear. Here we present an updated systematic review addressing the use of MT in paediatric patients, including three cases from our centre in Sydney, Australia. We have also completed an individual participant data (IPD) meta-analysis of clinical and angiographic outcomes based on these results.

Method Our systematic review and IPD meta-analysis was performed according to PRISMA-IPD (Preferred Reporting Items for Systematic Reviews and Meta-Analyses: Individual Participant Data) guidelines. Primary outcomes measures were change in NIHSS (National Institute of Health Stroke Scale) score following MT, and mRS (modified Rankin Scale) score at final reported follow-up. The secondary outcome measure was final angiographic result using the mTICI (modified Treatment in Cerebral Ischemia) scale.

Results MT resulted in good long-term neurological outcomes (mRS 0-2) in 60/67 cases (89.6\%;follow-up timing $\mu=4.1$ months: 95\%CI 2.9-5.3), good short-term neurological outcomes (reduction in NIHSS by 8 or more points or post-MT NIHSS of $0-1$ ) in $37 / 52$ cases $(71.2 \%)$, and successful recanalization (mTICI $2 \mathrm{~b} / 3$ ) in $57 / 67$ cases $(85.1 \%$ ).

Conclusions

In paediatric patients, MT is an effective treatment for ischaemic stroke due to LVO. In the absence of a dedicated prospective registry and with randomized control trails unfeasible, this report represents the best available evidence for the use of MT in the paediatric setting.

\section{THE IMPACT OF AGGRESSIVE BLOOD PRESSURE MANAGEMENT IN THE POST-THROMBOLYSIS SETTING}

${ }^{1}$ Bethan Harper*, ${ }^{2}$ Harry McNaughton, ${ }^{3}$ Anna Ranta. ${ }^{1}$ Neurology, Capital and Coast District Health Board and University of Otago, Wellington, New Zealand; ${ }^{2}$ Medical Research Institute of New Zealand, Wellington, New Zealand; ${ }^{3}$ Capital and Coast District Health Board and University of Otago, Wellington, Wellington, New Zealand

\subsection{6/jnnp-2019-anzan.13}

Introduction High blood pressure (BP) post-thrombolysis has been associated with an increased rate of bleeding and poorer outcome. We noted frequent BPs of $>180 \mathrm{mmHg}$ with a target of keeping $\mathrm{BP}<180$. We tested whether a more aggressive target of SBP $<160 \mathrm{mmHg}$ would result in fewer BP protocol violations.

Methods Patients were prospectively captured comparing patients thrombolysed during the 12 months before and 12 months following the introduction of a new more aggressive $\mathrm{BP}$ protocol, allowing for a 6 month transition period. Results were adjusted for baseline function and stroke severity using regression analysis.

Results Pre-protocol change 68 and post- 100 patients were thrombolysed. Baseline characteristics were similar between groups. There was a trend for a lower rate of SBPs $>180$ $\mathrm{mmHg}$ (adjusted OR 0.49; 95\% CI 0.31-1.1; $\mathrm{p}=0.097$ ) and a significantly higher rate of SBPs $<120 \mathrm{mmHg}$ (adjusted OR 3.06 ; 95\% CI $1.52-6.17 ; \mathrm{p}=0.002$ ) in the aggressive BP protocol group; although events of extreme SBPs (>200 and $<100 \mathrm{mmHg}$ ) were similar between groups. Favourable outcomes (mRS $=0-2)$ at 3 months were similar between groups (adjusted OR 1.27; 95\% CI $0.58-2.8 ; \mathrm{p}=0.56$ ) as was the rate of symptomatic haemorrhages (adjusted OR 1.26;95\% CI 0.28-5.7; $\mathrm{p}=0.76$ ). Model fit was improved by adding study group to the model.

Conclusions More aggressive post-thrombolysis BP management lowered the overall BP, but did not result in improved patient outcomes. Potential explanations include a small sample size, reduced cerebral perfusion off-setting reduced bleeding risk, or high BP being merely an epiphenomenon of worse outcome rather than causative.

\section{AVXS-101 GENE-REPLACEMENT THERAPY (GRT) IN PRESYMPTOMATIC SPINAL MUSCULAR ATROPHY (SMA): STUDY UPDATE}

${ }^{1}$ Michelle Farrar* ${ }^{*}$ Kathryn J Swoboda, ${ }^{3}$ Meredith Schultz, ${ }^{4}$ Hugh McMillan, ${ }^{5}$ Julie Parsons, ${ }^{6}$ lan E Alexander, ${ }^{3}$ Elaine Kernbauer, ${ }^{3}$ Marcia Farrow, ${ }^{3}$ Francis G Ogrinc, ${ }^{3}$ Douglas E Feltner, ${ }^{3}$ Bryan E McGill, ${ }^{3}$ Sidney A Spector, ${ }^{3}$ James L'Italien, ${ }^{3}$ Douglas M Sproule, ${ }^{7}$ Kevin A Strauss. ${ }^{1}$ Department of Neurology, Sydney Children's Hospital, Randwick, NSW, Australia; ${ }^{2}$ Department of Neurology, Massachusetts General Hospital, Boston, MA, USA; ${ }^{3}$ AveXis, Inc., Bannockburn, IL, USA; ${ }^{4}$ Department of Pediatrics, Canada Children's Hospital of Eastern Ontario, Ontario, Ottawa, Canada; ${ }^{5}$ Department of Neurology, Children's Hospital Colorado, Aurora, CO, USA; ${ }^{6}$ Gene Therapy Research Unit, Children's Medical Research Institute and The Children's Hospital at Westmead, Sydney, NSW, Australia; ${ }^{7}$ Clinic for Special Children, Strasburg, PA, USA

10.1136/jnnp-2019-anzan. 14

Introduction SMA is a neurodegenerative disease caused by biallelic deletion/mutation of the survival motor neuron 1 